\title{
Factors associated with early mortality after cervical spinal cord injury
}

\section{Jiang Shao, Wei Zhu, Xiongsheng Chen, Lianshun Jia, Dianwen Song, Xuhui Zhou, Wangjun Yan, Yong Zhang}

\author{
Department of Orthopaedics, Shanghai Changzheng Hospital, Shanghai, P.R. China
}

Background: A relatively high early mortality rate ( $<30$ days post-injury) for cervical spinal cord injury (SCI) has been observed.

Objective: To investigate this early mortality rate observed after cervical $\mathrm{SCl}$ and analyze the associated influential factors.

Methods: Medical records for 1163 patients with cervical SCI were reviewed, and the number of patients with early mortality was documented. Through logistic regression analysis, the effects of age, gender, occupation, cause of injury, severity of injury, highest involved spinal cord segment, nutritional condition during hospitalization, surgical treatment, tracheotomy, etc., on early mortality were assessed. Implementation of early treatment (i.e. surgery, tracheotomy, and nutritional support) and its effect on patient prognosis were also analyzed.

Results: Early mortality occurred in 109 of 1163 patients (9.4\%). Four factors affected the early mortality rate, including level and severity of $\mathrm{SCl}$, whether or not surgery was performed, the time interval between $\mathrm{SCl}$ and surgery, malnutrition, and tracheotomy. Patients with an American Spinal Injury Association grade of $\mathrm{A}$, a high cervical $\mathrm{SCl}$ (C1-C3), and/or no surgical intervention were statistically more likely to have early mortality $(P<0.001)$.

Conclusion: Severe cervical SCI, upper-level cervical cord injury, malnutrition, and inappropriate tracheotomy are risk factors for early mortality in patients with cervical SCl. Surgery can reduce early mortality. Early tracheotomy should be performed in patients with complete upper-level cervical SCl, but patients with incomplete cervical SCI or complete low-level cervical SCl should initially be treated surgically to maintain smooth airway flow.

Keywords: Mortality, Risk factors, Spinal cord injuries, Cervical, Malnutrition, Tracheotomy, Ventilation, Mechanical

\section{Introduction}

Cervical spinal cord injury (SCI) affects $2-3 \%$ of all trauma patients and accounts for $8.2 \%$ of all traumarelated deaths. ${ }^{1-3}$ Often associated with alterations in respiratory function secondary to disruption of innervation to the diaphragm and intercostal muscles and the need for mechanical ventilation, cervical SCI is associated with an unacceptably high-mortality rate. ${ }^{4}$ The majority of patients sustaining cervical SCI occur in young Caucasian men involved in motor vehicle accidents. ${ }^{5}$ The mechanisms of injury to the cervical spine include forced hyperextension, forced flexion, and compression. ${ }^{1}$ Cervical SCI can present with complete or

Correspondence to: Xiongsheng Chen, Department of Orthopaedics, Shanghai Changzheng Hospital, \#415 Feng Yang Road, Shanghai 200003, P.R. China. Email: chenxiongsheng@vip.sohu.com partial loss of all movements and/or sensation and be classified as either incomplete or complete based on the presence or absence of any residual voluntary movement or sensation below the level of the lesion.

Respiratory complications are cited as the primary cause of cervical SCI mortality; however, little is known about the contributing factors associated with early mortality in these patients. ${ }^{4}$ Therefore, a statistical analysis was performed on a large number of patients with cervical SCI 1 month post-injury in order to gain a better understanding of the main factors influencing early death in patients with cervical SCI. These data may reveal areas for improvement in the early treatment of these patients, and will either validate or refute current theories about the management of SCI, with the ultimate goal being reducing morbidity and mortality. 


\section{Materials and methods}

Medical records were reviewed of all 1187 patients hospitalized at Changzheng Hospital in Shanghai within 10 days after sustaining cervical SCI from January 1995 through December 2008. A total of 24 patients with associated abnormal vital signs caused by traumatic brain injury, thoracic and/or abdominal trauma, and severe injuries involving the pelvis and extremities (these patients had obvious abnormal vital signs or organ dysfunction, for example, pelvic fractures combined hemorrhagic shock, or extremity injury combined with crush syndrome or acute kidney failure) were excluded from further study. The following data were collected for the remaining 1163 patients: age, gender, occupation, cause of injury, American Spinal Injury Association (ASIA) grade, level of spinal injury, type of surgery (if any) performed, whether or not tracheotomy was performed, nutritional status, and prognosis. For many patients with high cervical SCI (at and above $\mathrm{C} 4$ level), tracheotomy surgery was often performed at 2-3 days after the injury for indications including severe difficulty breathing, acid-base balance disorders, and abnormal heart rate and blood pressure. The ASIA grade indicates the patient's functional impairment as a result of the SCI. ASIA grade A indicates complete SCI with loss of both motor and sensory function below the level of lesion, including in the sacral segments S4-S5. ASIA grades B-D indicate incomplete SCI, with some preservation of sensory and/or motor function below the neurologic level of injury. ${ }^{2}$ ASIA grade $\mathrm{E}$ indicates no impairment. There were no patients with ASIA grade $\mathrm{E}$ in the study. Prognosis was categorized as either early mortality (death within 30 days of the SCI) or alive $>30$ days post-injury. Assessment of nutritional status was based upon serial albumin levels checked every 3 days. A patient was classified as well-nourished if the albumin remained $>3.5 \mathrm{~g} / \mathrm{dl}$ or dropped below this level on only one occasion during the hospital stay. Patients with serum albumin $<3.5 \mathrm{~g} / \mathrm{dl}$ on more than one occasion were considered malnourished. This study was approved by the hospital's Institutional Review Board.

\section{Statistical analysis}

The continuous data with normal distribution (e.g. age) are summarized as mean \pm standard deviation, the continuous data not normally distributed (e.g. time to surgery) are summarized as median with inter-quartile range, while the difference in age between the patients with and without early mortality was compared by two-sample $t$-test, and the difference in time to surgery between the patients with and without early mortality was compared by Mann-Whitney test. Categorical variables are summarized as count and percentage, and their associations with early mortality were tested with Fisher's exact test. Univariate and multivariate logistic regression models were used for investigating the risk factors for early mortality and are presented as odds ratios (OR) and corresponding 95\% confidence intervals (CI). Dummy variables for categorical variables were created to add the information about them in the logistic regression model. Variance inflation factors (VIFs) were calculated to evaluate multicollinearity among the variables determined to be predictors of early mortality by univariate regression analysis. ${ }^{6}$ Variables with VIFs $>10$ were excluded from subsequent multivariate logistic regression analysis, then other variables were included in the multivariate logistic regression model by selection with the forward conditional stepwise method. ${ }^{7}$ All statistical assessments were two-sided using a significance level of 0.05. Statistical analyzes were performed by using SPSS 15.0 software package (SPSS Institute Inc., Chicago, IL, USA).

Among the seven variables of age, cause of injury, ASIA grade, segment of injury, cervical surgery, tracheotomy, and nutritional status that obtained the significance level of 0.05 in Table 3 for all 1163 patients, age and nutritional status were the first excluded from the multivariate analysis due to multicollinearity (VIFs $>10$ ); then cause of injury was excluded by the forward conditional stepwise method because it was not an independent factor. Therefore, only ASIA grade, segment of injury, cervical surgery, and tracheotomy were included in the multivariate analysis.

Among the five variables of cause of injury, ASIA score, tracheotomy, nutritional status, and time to surgery that obtainedlthe significance level of 0.05 in Table 4 for 901 patients who were treated with surgery, no variable was excluded from the multivariate analysis due to multicollinearity, but cause of injury was excluded from the multivariate analysis by the forward conditional stepwise method because it was not an independent factor. Therefore, only ASIA score, tracheotomy, nutritional status, and time to surgery were included in the multivariate analysis.

\section{Results}

\section{Patient demographics}

A total of 1163 patients (including 974 men and 189 women) who sustained cervical SCI from January 1995 through December 2008 were included in this study. The mean age for all the patients in the study 
was $48.3 \pm 10.9$ years (range 17-86 years). Among the 1163 patients, $109(9.4 \%)$ suffered early mortality. Causes of death included respiratory failure with concomitant hypoxemia and acid-base disorders resulting in cardiac arrhythmia and cardiac arrest $(n=95)$; multiple organ failure $(n=6)$, sudden cardiac arrest $(n=5)$, and acute gastrointestinal hemorrhage $(n=3)$. The remaining 1054 patients survived $>30$ days post-injury. The patients with early mortality were significantly older ( $48.59 \pm 13.96$ versus $44.85 \pm 13.78$ years, $P=0.007$ ). This indicated that older patients with cervical SCI were more likely to suffer early mortality. No significant differences in gender or occupation were identified between groups. Those patients with cervical SCI related to traffic accidents were also statistically more likely to survive beyond 30 days $(P=0.008)$ (Table 1$)$.

\section{Spinal cord injury}

Patients with an ASIA grade of A, a high cervical SCI $(\mathrm{C} 1-3)$, and/or no surgical intervention were significantly more likely to suffer early mortality $(P<$ $0.001)$. The vast majority $(84.4 \%)$ of patients experiencing early mortality had an ASIA grade of A; however, only $29.4 \%$ of patients surviving $>30$ days had an ASIA grade of A $(P<0.001)$. Among those patients with early mortality, $20.2 \%$ sustained $\mathrm{C} 1-\mathrm{C} 3$ SCI, $55.0 \%$ sustained C4-C5 SCI, and 24.8\% sustained C6-C8 SCI. In the group surviving $>30$ days, more patients experienced $\mathrm{C} 6-\mathrm{C} 8 \mathrm{SCI}$, and fewer patients experienced $\mathrm{C} 1-\mathrm{C} 3 \mathrm{SCI}$ than those in the early mortality group $(9.2 \% \quad \mathrm{C} 1-\mathrm{C} 3 ; 49.1 \% \mathrm{C} 4-\mathrm{C} 5 ; 41.7 \%$ C6-C8). A C1-C3 SCI was significantly associated with early mortality $(P<0.001)$.

Table 1 Associations between baseline conditions and early mortality

\begin{tabular}{|c|c|c|c|}
\hline & \multicolumn{2}{|c|}{ Prognosis status } & \multirow[b]{2}{*}{$\boldsymbol{P}$-value } \\
\hline & $\begin{array}{l}\text { Early mortality } \\
\qquad(n=109)\end{array}$ & $\begin{array}{l}\text { Alive }>30 \text { days } \\
\quad(n=1054)\end{array}$ & \\
\hline Age (year) & $48.59 \pm 13.96$ & $44.85 \pm 13.78$ & $0.007^{*}$ \\
\hline \multicolumn{4}{|l|}{ Gender } \\
\hline Male & $96(88.1 \%)$ & $878(83.3 \%)$ & 0.222 \\
\hline Female & $13(11.9 \%)$ & $176(16.7 \%)$ & - \\
\hline \multicolumn{4}{|l|}{ Occupation } \\
\hline Blue collar & $74(67.9 \%)$ & $712(67.6 \%)$ & 1.000 \\
\hline White collar & $35(32.1 \%)$ & $342(32.4 \%)$ & - \\
\hline \multicolumn{4}{|l|}{ Cause of injury } \\
\hline Traffic accident & $29(27.1 \%)$ & $422(41.5 \%)$ & $0.008^{*}$ \\
\hline Fall & 38 (35.5\%) & 258 (25.4\%) & - \\
\hline Others & $40(37.4 \%)$ & $336(33.1 \%)$ & - \\
\hline \multicolumn{4}{|l|}{ ASIA grade } \\
\hline$A$ & $92(84.4 \%)$ & $310(29.4 \%)$ & $<0.001^{*}$ \\
\hline B & $9(8.3 \%)$ & $112(10.6 \%)$ & - \\
\hline C & $4(3.7 \%)$ & $222(21.1 \%)$ & - \\
\hline D & $4(3.7 \%)$ & 410 (38.9\%) & - \\
\hline \multicolumn{4}{|l|}{ Segment of injury } \\
\hline C1-3 & $22(20.2 \%)$ & $97(9.2 \%)$ & $<0.001^{*}$ \\
\hline C4-5 & $60(55.0 \%)$ & $517(49.1 \%)$ & - \\
\hline C6-8 & $27(24.8 \%)$ & $440(41.7 \%)$ & - \\
\hline \multicolumn{4}{|l|}{ Cervical surgery } \\
\hline No surgery & $84(77.1 \%)$ & $178(16.9 \%)$ & $<0.001^{*}$ \\
\hline Anterior & $17(15.6 \%)$ & $672(63.8 \%)$ & - \\
\hline Posterior & $4(3.7 \%)$ & $120(11.4 \%)$ & - \\
\hline Anterior-posterior & $4(3.7 \%)$ & $84(8.0 \%)$ & - \\
\hline \multicolumn{4}{|l|}{ Tracheotomy } \\
\hline Yes & 47 (43.1\%) & 77 (7.3\%) & $<0.001^{*}$ \\
\hline No & $62(56.9 \%)$ & $977(92.7 \%)$ & - \\
\hline \multicolumn{4}{|l|}{ Nutritional status } \\
\hline Malnutrition & $87(79.8 \%)$ & $282(26.8 \%)$ & $<0.001^{*}$ \\
\hline Normal & $22(20.2 \%)$ & 772 (73.2\%) & - \\
\hline Time to surgery** (days) & $8(4-11)$ & $9(6-15)$ & $0.034^{*}$ \\
\hline
\end{tabular}

All variables were presented by count (percentage) except for age presented as mean \pm SD, time to surgery presented as median with range.

${ }^{\star} P<0.05$ indicates a significant association between the variable and early mortality.

${ }^{* *}$ Nine hundred and one patients treated with surgery were analyzed. 


\section{Management of SCI}

More than $75 \%$ (77.1\%) of patients with early mortality did not receive any surgical intervention, while $83 \%$ of patients surviving $>30$ days did receive an anterior, posterior, or anterior-posterior surgical intervention. The absence of surgical intervention was significantly associated with early mortality $(P<0.001)$. Forty-seven $(37.9 \%)$ out of 124 patients with tracheotomy surgery died within 30 days, while only $62(6.0 \%)$ out of 1039 patients without tracheotomy surgery died within 30 days (Table 1). For the 402 patients with complete cervical SCI, $40(42.1 \%)$ out of 95 patients with tracheotomy surgery died within 30 days while only 52 (16.9\%) out of 307 patients without tracheotomy surgery died within 30 days. Similar results were observed in those injured at the segments of $\mathrm{C} 4-\mathrm{C} 5$ (18.9 versus $52.0 \%, P<0.001)$ or $\mathrm{C} 6-\mathrm{C} 8$ ( 7.3 versus $27.5 \%, P=0.001)$. But a contrasting result was observed in those injured at the segments of $\mathrm{C} 1-\mathrm{C} 3$; the early mortality rate for those patients with tracheotomy was significantly lower than for those without tracheotomy (60.0 versus $100 \%, P=0.048$ ) (Table 2). Among all patients, $9.4 \%$ died within 30 days and $7 \%$ of patients surviving $>30$ days required tracheotomy. Tracheotomy surgery was significantly associated with early mortality $(P<0.001)$. Furthermore, nearly $80 \%$ of patients in the early mortality group were nutritionally malnourished compared to $27 \%$ of the patients surviving $>30$ days $(P<0.001)$ (Table 1$)$.

\section{Risk factors for early mortality}

The risk factors for early mortality in univariate and multivariate logistic regression models are summarized in Table 3. Seven variables obtained statistical significance $(P<0.05)$ in the univariate logistic regression models. Age and nutrition had VIFs $>10$ (indicating collinearity) and were not included in multivariate regression analysis. After model selection, cause of injury was excluded from the multivariate logistic regression model; hence ASIA grade, segment of injury, cervical surgery, and tracheotomy were included in the multivariate logistic regression model.

In the multivariate logistic regression model for all patients (Table 3), compared with patients who had ASIA grades of $\mathrm{D}$, patients were more likely to have early mortality if they had grades of A (64.73 OR [95\% CI, 20.66-202.78]), B (15.08 OR [95\% CI, 3.86-58.86]), or C (4.64 OR [95\% CI, 1.07-20.07]) $(P<0.001, P<0.001, P=0.040$, respectively).

Patients with $\mathrm{C} 1-\mathrm{C} 3$ and $\mathrm{C} 4-\mathrm{C} 5 \mathrm{SCI}$ demonstrated a higher risk of early mortality when compared to those patients with C6-C8 SCI $(5.03$ OR $[95 \%$ CI, 1.84-13.73] for $\mathrm{C} 1-\mathrm{C} 3 \mathrm{SCI} ; 2.14$ OR $[95 \% \mathrm{CI}$, 1.13-4.06] for C4-C5 SCI $(P=0.002, P=0.020)$.

Compared to those not treated with surgery, patients treated with anterior, posterior, or anterior-posterior surgery were less likely to experience early mortality (0.03 OR [95\% CI, 0.02-0.06], 0.05 OR [95\% CI, $0.02-0.18$ ], and 0.04 OR [95\% CI, 0.01-0.14]) than those treated with anterior, posterior, and anteriorposterior surgery, respectively. Patients who underwent tracheotomy had a significantly higher risk of early mortality than those who did not undergo tracheotomy (3.56 OR [95\% CI, 1.86-6.81]; $P<0.001$ ).

In addition, the risk factors for early mortality for the 901 patients treated with surgery are summarized in Table 4. Five variables obtained statistical significance $(P<0.05)$ in the univariate logistic regression models. No variables among the five were excluded due to multicollinearity, so that all of them were selected by the forward conditional method. After model selection, cause of injury was excluded from the multivariate logistic regression model; hence, ASIA grade, tracheotomy, nutritional status, and time to surgery were included in the multivariate logistic regression model.

In the multivariate logistic regression model for patients treated with surgery (Table 4), patients who had ASIA grades of A were more likely to have early mortality than those patients who had ASIA grades of

Table 2 The early mortality rate for complete cervical SCI patients (ASIA score A, $n=402$ ) with or without tracheotomy

\begin{tabular}{|c|c|c|c|c|}
\hline Segment of injury & Survival status & $\begin{array}{l}\text { With tracheotomy } \\
\qquad(n=95)\end{array}$ & $\begin{array}{l}\text { Without tracheotomy } \\
\qquad(n=307)\end{array}$ & $\boldsymbol{P}$-value \\
\hline \multirow[t]{2}{*}{ C1-3 } & Early mortality & $3(60.0 \%)$ & $16(100.0 \%)$ & $0.048^{*}$ \\
\hline & Alive more than 30 days & $2(40.0 \%)$ & $0(0.0 \%)$ & - \\
\hline \multirow[t]{2}{*}{ C4-5 } & Early mortality & $26(52.0 \%)$ & $24(18.9 \%)$ & $<0.001^{*}$ \\
\hline & Alive more than 30 days & $24(48.0 \%)$ & $103(81.1 \%)$ & - \\
\hline \multirow[t]{2}{*}{ C6-8 } & Early mortality & $11(27.5 \%)$ & $12(7.3 \%)$ & $0.001^{*}$ \\
\hline & Alive more than 30 days & 29 (72.5\%) & $152(92.7 \%)$ & - \\
\hline \multirow[t]{2}{*}{ Total } & Early mortality & $40(42.1 \%)$ & $52(16.9 \%)$ & $<0.001^{*}$ \\
\hline & Alive more than 30 days & $55(57.9 \%)$ & $255(83.1 \%)$ & - \\
\hline
\end{tabular}

${ }^{\star} P<0.05$ indicates a significant difference was observed in the early mortality rate between those with and without tracheotomy. 
Table 3 The univariate and multivariate logistic regression models for early mortality ( $n=1163$ for all patients)

\begin{tabular}{|c|c|c|c|c|}
\hline & \multicolumn{2}{|c|}{ Univariate } & \multicolumn{2}{|c|}{ Multivariate } \\
\hline & Odds ratio $(95 \% \mathrm{Cl})$ & $\boldsymbol{P}$-value & Odds ratio $(95 \% \mathrm{Cl})$ & $\boldsymbol{P}$-value \\
\hline Age & $1.02(1.01,1.03)$ & $0.007^{\star}$ & - & - \\
\hline \multicolumn{5}{|l|}{ Gender } \\
\hline Male & $1.48(0.81,2.70)$ & 0.201 & - & - \\
\hline Female & - & - & - & - \\
\hline \multicolumn{5}{|l|}{ Occupation } \\
\hline White collar & $0.98(0.65,1.50)$ & 0.943 & - & - \\
\hline Blue collar & - & - & - & - \\
\hline \multicolumn{5}{|l|}{ Cause of injury } \\
\hline Traffic accident & - & - & - & - \\
\hline Fall & $2.14(1.29,3.56)$ & $0.003^{*}$ & - & - \\
\hline Others & $1.73(1.05,2.85)$ & $0.031^{*}$ & - & - \\
\hline \multicolumn{5}{|l|}{ ASIA grade } \\
\hline$A$ & $30.42(11.06,83.68)$ & $<0.001^{\star}$ & $64.73(20.66,202.78)$ & $<0.001^{\star}$ \\
\hline B & $8.24(2.49,27.24)$ & $<0.001^{*}$ & $15.08(3.86,58.86)$ & $<0.001^{*}$ \\
\hline C & $1.85(0.46,7.46)$ & 0.389 & $4.64(1.07,20.07)$ & $0.040^{*}$ \\
\hline $\mathrm{D}$ & - & - & - & - \\
\hline \multicolumn{5}{|l|}{ Segment of injury } \\
\hline C1-3 & $3.70(2.02,6.76)$ & $<0.001^{*}$ & $5.03(1.84,13.73)$ & $0.002^{*}$ \\
\hline C4-5 & $1.89(1.18,3.03)$ & $0.008^{*}$ & $2.14(1.13,4.06)$ & $0.020^{*}$ \\
\hline C6-8 & - & - & - & - \\
\hline \multicolumn{5}{|l|}{ Cervical surgery } \\
\hline No surgery & - & - & - & - \\
\hline Anterior & $0.05(0.03,0.09)$ & $<0.001^{*}$ & $0.03(0.02,0.06)$ & $<0.001^{*}$ \\
\hline Posterior & $0.07(0.03,0.20)$ & $<0.001^{*}$ & $0.05(0.02,0.18)$ & $<0.001^{*}$ \\
\hline Anterior-posterior & $0.10(0.04,0.28)$ & $<0.001^{*}$ & $0.04(0.01,0.14)$ & $<0.001^{\star}$ \\
\hline \multicolumn{5}{|l|}{ Tracheotomy } \\
\hline Yes & $9.62(6.17,15.00)$ & $<0.001^{*}$ & $3.56(1.86,6.81)$ & $<0.001^{*}$ \\
\hline No & - & - & - & - \\
\hline \multicolumn{5}{|l|}{ Nutritional status } \\
\hline Malnutrition & $10.83(6.65,17.62)$ & $<0.001^{*}$ & - & - \\
\hline Normal & - & - & - & - \\
\hline
\end{tabular}

${ }^{\star} P<0.05$ indicated that the $95 \% \mathrm{Cl}$ for odds ratio did not include 1 .

-, Reference category.

$P$-value $=0.436$ by Hosmer and Lemeshow test implies an acceptable goodness of fit for the multivariate model.

D (15.01 OR [95\% CI, 1.90-118.94], $P=0.010)$. Patients who underwent tracheotomy had a significantly higher risk of early mortality than those who did not undergo tracheotomy (3.98 OR [95\% CI, 1.47-10.80]; $P=0.007$ ). Patients with malnutrition had a significantly higher risk of early mortality than those who had normal nutritional status $(9.45$ OR $[95 \%$ CI, 3.53-25.30]; $P<0.001)$. The risk of mortality slightly decreased every day as time to surgery increased $(0.92$ OR [0.85-0.999], $P=0.047$ ).

\section{Discussion}

This study showed that the factors influencing the early prognosis of patients with cervical SCI included both pre-determined, objective factors (severity of SCI and level of SCI), as well as potentially modifiable factors (surgical intervention and airway management).

Complete, higher levels of cervical SCI have been previously associated with early mortality. ${ }^{8}$ Our study findings are in keeping with those previously reported.
Complete cervical SCI at or above the $\mathrm{C} 3$ level disrupts innervation to the diaphragm and the intercostal muscles. These patients require immediate mechanical ventilation and often die immediately after the SCI secondary to respiratory failure and/or cardiovascular dysfunction. ${ }^{9}$ In patients with a complete cervical SCI below the $\mathrm{C} 4$ level, early mortality decreases significantly compared to the levels above C3. However, since there are some upper motor neurons of the phrenic nerves in the cervical spinal cord at the $\mathrm{C} 4$ level, a complete cervical SCI at this level can affect the motor function of the diaphragm to some extent, and the patient may still be susceptible to early death from respiratory failure. Mortality rates continue to decline as the involved cervical spine level decreases. In patients with cervical SCI below the C4 level, the function of the diaphragm remains intact despite complete functional loss of the intercostal muscles. Most patients can still maintain adequate pulmonary ventilation through diaphragm compensation. 
Table 4 The univariate and multivariate logistic regression models for early mortality $(n=901$, those patients who treated with surgery)

\begin{tabular}{|c|c|c|c|c|}
\hline & \multicolumn{2}{|c|}{ Univariate } & \multicolumn{2}{|c|}{ Multivariate } \\
\hline & Odds ratio $(95 \% \mathrm{Cl})$ & $\boldsymbol{P}$-value & Odds ratio $(95 \% \mathrm{Cl})$ & $\boldsymbol{P}$-value \\
\hline Age & $1.02(0.99,1.05)$ & 0.292 & - & - \\
\hline \multicolumn{5}{|l|}{ Gender } \\
\hline Male & $4.80(0.64,35.76)$ & 0.126 & - & - \\
\hline Female & - & - & - & - \\
\hline \multicolumn{5}{|l|}{ Occupation } \\
\hline White collar & $1.30(0.57,2.99)$ & 0.530 & - & - \\
\hline Blue collar & - & - & - & - \\
\hline \multicolumn{5}{|l|}{ Cause of injury } \\
\hline Traffic accident & - & - & - & - \\
\hline Fall & $3.16(1.07,9.36)$ & $0.038^{*}$ & - & - \\
\hline Others & $2.15(0.70,6.64)$ & 0.184 & - & - \\
\hline \multicolumn{5}{|l|}{ ASIA score } \\
\hline A & $22.34(2.99,167.18)$ & $0.002^{*}$ & $15.01(1.90,118.94)$ & $0.010^{*}$ \\
\hline$B$ & $5.88(0.53,65.56)$ & 0.150 & $4.03(0.34,47.00)$ & 0.267 \\
\hline C & $1.56(0.10,25.13)$ & 0.753 & $1.35(0.08,21.97)$ & 0.833 \\
\hline D & - & - & - & - \\
\hline \multicolumn{5}{|l|}{ Segment of injury } \\
\hline C1-3 & $0.71(0.09,5.77)$ & 0.749 & - & - \\
\hline C4-5 & $1.72(0.73,4.07)$ & 0.214 & - & - \\
\hline C6-8 & - & - & - & - \\
\hline \multicolumn{5}{|l|}{ Cervical surgery } \\
\hline Anterior & - & - & - & - \\
\hline Posterior & $0.53(0.17,1.62)$ & 0.265 & - & - \\
\hline Anterior-posterior & $0.70(0.17,2.88)$ & 0.621 & - & - \\
\hline \multicolumn{5}{|l|}{ Tracheotomy } \\
\hline Yes & $6.58(2.80,15.44)$ & $<0.001^{*}$ & $3.98(1.47,10.80)$ & $0.007^{*}$ \\
\hline No & - & - & - & - \\
\hline \multicolumn{5}{|l|}{ Nutritional status } \\
\hline Malnutrition & $8.54(3.37,21.64)$ & $<0.001^{*}$ & $9.45(3.53,25.30)$ & $<0.001^{\star}$ \\
\hline Normal & - & - & - & - \\
\hline Time to surgery & $0.92(0.85,0.99)$ & $0.019^{*}$ & $0.92(0.85,0.999)$ & $0.047^{\star}$ \\
\hline
\end{tabular}

${ }^{*} P<0.05$ indicated that the $95 \% \mathrm{Cl}$ for odds ratio did not include 1 .

-, Reference category.

$P$-value $=0.200$ by Hosmer and Lemeshow test implies an acceptable goodness of fit for the multivariate model.

Incomplete cervical SCI are associated with lower early mortality rates. Alander et al. reported that among patients $>50$ years of age, incomplete cervical SCI were associated with a better rate of patients being able to return home and resumption of a functional lifestyle. ${ }^{10}$ In these patients, the diaphragmatic function and some remnant innervating intercostal muscles contribute to maintaining the respiratory drive. A cough reflex persists, sympathetic function remains intact, and bronchospasm is an infrequent complication. However, vigilance is required for other complications, such as cardiopulmonary arrest and upper gastrointestinal bleeding.

Because of the lack of a definitive cure, special attention must be paid to both the prevention of additional damage to the spinal cord and the treatment of existing problems subsequent to the SCI. Surgical intervention may be considered to stabilize the cervical spine and prevent secondary SCI. However, controversy exists as to whether surgery actually improves patient survival in cases of severe SCI. ${ }^{11-13}$ Approximately 25\% of patients in our study did not receive surgical intervention, and the results suggest that early mortality was significantly reduced in those patients undergoing surgery. Surgical intervention may allow more motion of the head and extremities and facilitate an improvement in pulmonary ventilation, which may subsequently increase the early survival rate of patients with severe cervical SCI. Therefore, patients with cervical SCI who have cord compression and cervical spinal instability should receive surgical intervention, even in the presence of poor ventilation, dyspnea, and other symptoms of respiratory failure.

The use of early and timely tracheotomy has been shown to decrease the mortality in patients with cervical SCI. ${ }^{14}$ Additionally, Harrop et al. suggested early tracheotomy facilitates weaning from mechanical ventilation, decreases intensive care unit stay, and ultimately reduces overall hospital costs. ${ }^{1}$ However, our study showed that early mortality was significantly 
higher in patients who underwent tracheotomy; furthermore, it was much higher in patients with complete cervical SCI who underwent tracheotomy than in those patients who had the same extent of injury, but did not undergo tracheotomy (Table 2). Several possible explanations exist. First of all, patients who underwent tracheotomy often had more severe injuries and sustained higher levels of cervical SCI. They were more susceptible not only to respiratory failure, but also to cardiac arrest and multiple organ failure. The invasive nature of tracheotomy surgery may add additional physiological stress to the already fragile patient. Tracheotomy breaches the natural barrier between the lung and the outside environment, significantly increasing the probability of infection. Additionally, it is possible that the indications for and timing of tracheotomy were not controlled properly. In our study, the rate of tracheotomy was unexpectedly quite low in patients with high-level cervical SCI (at and above C4 level). Tracheotomy was often performed emergently after the onset of severe respiratory failure (three SCI C1 + C2 patients, Table 2), and these three patients died immediately after the tracheotomy. For patients with low-level cervical SCI, airway management (e.g. management of secretions with general suction or bronchoscope) was not optimized. The onset of dyspnea in these patients led to unnecessary tracheotomies in some patients. In fact, clinical practice in recent years has proven that most patients with low-level cervical SCI can maintain normal spontaneous breathing without the need for tracheotomy.

Tracheotomy should be carried out as soon as possible for patients with severe cervical SCI at or above the $\mathrm{C} 3$ level because of the inevitable diaphragmatic paralysis; however, tracheotomy is usually unnecessary for the patients with cervical SCI at or below C6 level because these patients have normal diaphragmatic function and only require management of airway secretions. For the patients with severe SCI at the $\mathrm{C} 4$ and $\mathrm{C} 5$ levels, close observation is required. ${ }^{15,16}$ Interestingly, in the early mortality group, the patients had a slightly shorter time to surgery. The difference was not large. Maybe these patients were believed to have more serious disease and were sent to surgery more quickly.

Several limitations are inherent to this study. Cervical SCI are often associated with significant trauma, and patients are expected to have sustained multiple injuries. The selection criteria for this study excluded patients with traumatic brain injury, thoracic and/or abdominal trauma, and severe injuries involving the pelvis and extremities. Inclusion of these patients may have altered the significance of the mortality data. There was no mention as to the standardization of care received by these patients, and therefore, the possibility exists that some patients received treatment (e.g. corticosteroids), while others did not. Some factors such as sepsis, chest infection, etc., which are important variables for the prognosis of cervical SCI, were not included in this study. However, the current project mainly focused on the effects of normal condition, injury status, and major treatments on early prognosis of patients with cervical spinal cord injury. Factors such as sepsis, chest infection, hypoxemia, and ventilatory failure are all possible complications after cervical spinal cord injury. These factors and complications surely had effects on prognosis, but they were not the observed factors in this study as early predictive factors for the prognosis of cervical SCI. Furthermore, nearly $85 \%$ of the patients were male. Although this may accurately reflect the true incidence of SCI, further study is warranted to verify the validity of the results as they pertain to both sexes.

\section{Conclusion}

Our findings indicate that severe cervical SCI, upperlevel cervical cord injury, and malnutrition are risk factors for early mortality in patients with cervical SCI. Surgical intervention may reduce early mortality in patients with cervical SCI. Tracheotomy should be performed immediately for patients with high-level cervical SCI; however, patients with incomplete cervical SCI or complete low-level cervical SCI should initially be treated surgically to maintain smooth airway flow.

\section{References}

1 Harrop JS, Sharan AD, Scheid EH Jr, Vaccaro AR, Przybylski GJ. Tracheostomy placement in patients with complete cervical SCI: American Spinal Injury Association Grade A. J Neurosurg 2004; 100(1 Suppl Spine):20-3.

2 Kang SW, Shin JC, Park CI, Moon JH, Rha DW, Cho DH. Relationship between inspiratory muscle strength and cough capacity in cervical spinal cord injured patients. Spinal Cord 2006;44(4):242-8.

3 Shackford SR, Mackersie RC, Holbrook TL, Davis JW, Hollingsworth-Fridlund P, Hoyt DB, et al. The epidemiology of traumatic death: a population-based analysis. Arch Surg 1993; 128(5):571-5.

4 Frankenfeld D. Energy expenditure and protein requirements after traumatic injury. Nutr Clin Pract 2006;21(5):430-7.

5 National SCI Statistical Center SCI. Facts and figures at a glance. J Spinal Cord Med 2005;28(4):379-80.

6 Gordon RA. Citation-classic - issues in multiple regression. Curr Contents Social Behav Sci 1987;7(36):18.

7 Kutner M, Nachtsheim C, Neter J. Applied linear regression models. 4th ed. New York: McGraw-Hill Irwin; 2004.

8 Lemons VR, Wagner FC Jr. Respiratory complications after cervical spinal cord injury. Spine (Phila Pa 1976) 1994;19(20): 2315-20.

9 Durga P, Sahu BP, Mantha S, Ramachandran G. Development and validation of predictors of respiratory insufficiency and 
mortality scores: simple bedside additive scores for prediction of ventilation and in-hospital mortality in acute cervical spine injury. Anesth Analg 2010;110(1):134 40.

10 Alander DH, Andreychik DA, Stauffer ES. Early outcome in cervical spinal cord injured patients older than 50 years of age. Spine (Phila Pa 1976) 1994;19(20):2299-301.

11 Berlly M, Shem K. Respiratory management during the first five days after spinal cord injury. J Spinal Cord Med 2007;30(4): 309-18.

12 Hassid VJ, Schinco MA, Tepas JJ, Griffen MM, Murphy TL, Frykberg ER, et al. Definitive establishment of airway control is critical for optimal outcome in lower cervical spinal cord injury. J Trauma 2008;65(6):1328-32.
13 Vaccaro AR, Daugherty RJ, Sheehan TP, Dante SJ, Cotler JM, Balderston RA, et al. Neurologic outcome of early versus late surgery for cervical spinal cord injury. Spine (Phila Pa 1976) 1997;22(22):2609-13.

14 DeVivo MJ, Kartus PL, Rutt RD, Stover SL, Fine PR. The influence of age at time of spinal cord injury on rehabilitation outcome. Arch Neurol 1990;47(6):687-91.

15 Farmer J, Vaccaro A, Albert TJ, Malone S, Balderston RA, Cotler JM. Neurologic deterioration after cervical spinal cord injury. J Spinal Disord 1998;11(3):192-6.

16 Samsa GP, Patrick CH, Feussner JR. Long-term survival of veterans with traumatic spinal cord injury. Arch Neurol 1993;50(9): $909-14$. 